\title{
BMJ Open Protocol for a systematic review and meta-analysis of culturally adapted internet- and mobile-based health promotion interventions
}

\author{
Sumeyye Balci (D , ${ }^{1}$ Kerstin Spanhel, ${ }^{2}$ Lasse Sander, ${ }^{2}$ Harald Baumeister ${ }^{1}$
}

To cite: Balci S, Spanhel K, Sander L, et al. Protocol for a systematic review and meta-analysis of culturally adapted internet- and mobilebased health promotion interventions. BMJ Open 2020;10:e037698. doi:10.1136/ bmjopen-2020-037698

- Prepublication history and additional material for this paper is available online. To view these files, please visit the journal online (http://dx.doi.org/10. 1136/bmjopen-2020-037698)

Received 12 February 2020 Revised 10 July 2020 Accepted 02 October 2020

\section{Check for updates}

(c) Author(s) (or their employer(s)) 2020. Re-use permitted under CC BY-NC. No commercial re-use. See rights and permissions. Published by BMJ.

${ }^{1}$ Department of Clinical Psychology and Psychotherapy, Institute of Psychology and Education, University of UIm, Ulm, Germany

${ }^{2}$ Department of Rehabilitation Psychology and Psychotherapy, Institute of Psychology, AlbertLudwigs-Universitat Freiburg, Freiburg im Breisgau, BadenWürttemberg, Germany

Correspondence to

Sumeyye Balci;

sumeyye.balci@uni-ulm.de

\section{ABSTRACT}

Introduction High rates of immigration pose challenges for the healthcare systems of many countries to offer high-quality care to diverse populations. Advancing health interventions with incorporating the cultural background of diverse populations can be helpful to overcome this challenge. First studies suggest that culturally diverse populations might benefit from culturally adapted internetbased and mobile-based interventions (IMI) to promote health behaviours. However, the effectiveness of culturally adapted IMls for health promotion interventions has not been evaluated systematically. Therefore, the aim of this review is to assess the effectiveness of culturally adapted IMls regarding health promotion. Additionally, the cultural adaptation features of these interventions will be outlined. Methods and analysis Randomised controlled trials (RCTs) investigating the effectiveness of culturally adapted IMls to promote health behaviours in the field of healthy eating, smoking cessation, alcohol consumption, physical activity and sexual health behaviour will be identified via a systematic search of the databases MEDLINE, Embase, PsycINF0, CENTRAL. The preliminary search has been conducted on the 26 August 2019 and will be updated in the process. Data will be pooled meta-analytically in case of at least three included studies reporting on the same outcome. Moreover, a narrative synthesis of the included studies will be conducted. The risk of bias will be assessed using the Cochrane Collaboration's tool for the Quality Assessment of RCTs V. 2.0. Publication bias will be assessed using funnel plots.

Ethics and dissemination Ethical approval is not required for this study. The results of this study will be published in a peer-reviewed international journal. PROSPERO registration number PROSPERO; CRD42020152939

\section{INTRODUCTION}

Non-communicable diseases (NCDs) accounted for $71 \%$ of the global deaths in 2016. ${ }^{1}$ NCDs are also a major barrier in the reduction of the total burden of these diseases and achieving good health for all people. ${ }^{2}$ Minority populations suffer from NCDs more than the general population. ${ }^{3}$ Furthermore, compared with non-immigrant populations, immigrants show a higher prevalence of
Strengths and limitations of this study

- This is the first comprehensive systematic review of culturally adapted internet-based and mobile-based health promotion interventions.

- This review only includes randomised controlled trial studies to maintain a high methodological standard.

- This review covers the internet-based and mobilebased health promotion interventions in the areas of healthy eating, physical activity, sexual health behaviour, smoking cessation and alcohol consumption.

- Our search yielded only studies written in English, which might be indicative of a selective reporting bias.

- We are expecting a substantial heterogeneity in study designs and outcomes which might challenge firm conclusions.

diabetes, cardiovascular diseases (CVDs), HIV/AIDS ${ }^{4}$ and high blood pressure. ${ }^{56}$ The burden and prevalence of NCDs are on the rise in low-income countries as well as in middle-income and high-income countries. ${ }^{7}$ Furthermore, the risk is higher for people with a migration background living in middleincome and high-income countries. ${ }^{3-10}$

One approach to preventing NCDs and improving peoples health constitutes health promotion interventions. ${ }^{11}$ Health promotion interventions can modify behavioural risk factors by targeting healthy eating, exercising, ${ }^{12}$ not smoking, sexual health behaviour, namely condom use and HIV testing, and avoiding the harmful use of alcohol. ${ }^{13-15}$ These behaviours are related to morbidity and mortality globally ${ }^{16}$ and promoted by WHO to decrease the chances of developing NCD and to keep a healthy life. ${ }^{17} 18$ Additionally, socioeconomic factors and migration itself play an important role in accessing and benefiting from healthcare interventions. ${ }^{19} 20$ The access of immigrants and minority populations to health services 
can be hindered owing to several factors such as the health beliefs of the respective groups, language and cultural barriers, low health literacy, perceived stigma and limited financial means. ${ }^{21}$ To tackle the problems related to the accessibility of health services for immigrants and minority populations and to decrease the risk of developing NCDs, it might be essential to offer culturally sensitive health behaviour interventions to assure a more relevant and appealing practice to culturally diverse populations. ${ }^{322} 23$

An approach to making interventions more suitable for culturally diverse groups is to adapt existing interventions for the target group, which is called cultural adaptation. Cultural adaptation refers to systematic modifications of evidence-based practice by taking into account language, culture, and the context to make the intervention more suitable with the target group's cultural patterns, meanings and values. ${ }^{24}$ To guarantee the scientific excellence of the research and guide future researchers with the adaptation of interventions regarding public health, various frameworks for adaptations have been presented. A review identified 13 frameworks and eight common steps to adapt an intervention, among others: assessing the population of interest, understanding the original intervention and deciding on the aspects to adapt, the last two being the most commonly illustrated steps. ${ }^{25}$ More frameworks specifically addressing cultural adaptations are Ecological Validity ${ }^{26}$; Cultural Sensitivity Framework $^{27}$; Tool Kit of Adaptation Approaches ${ }^{28}$; Intervention mapping framework ${ }^{29}$; Cultural Accommodation Model. ${ }^{30}$ However, these frameworks were developed for face-to-face interventions.

Whereas face-to-face interventions are widely used, advances in internet technology and its increasing availability all around the world lead researchers to use this source to offer health behavioural change interventions digitally. ${ }^{31}$ Internet-based and mobile-based interventions (IMIs) are used to enhance health behaviour promotion and result in promising positive effects for general and minority populations. ${ }^{32-35}$ According to previous reviews, IMIs concerning health promotion are potentially effective in altering lifestyle factors and sporting medical treatments, ${ }^{35} 36$ health behavioural change, ${ }^{32} 34$ losing weight, ${ }^{33}{ }^{37} \mathrm{HIV}$ prevention, ${ }^{38} \mathrm{CVD}$ prevention, ${ }^{39}$ smoking cessation ${ }^{40}$ and physical activity. ${ }^{41}$ Furthermore, IMIs have been culturally adapted in order to reach culturally diverse populations. For example, culturally adapted IMIs yielded beneficial results for individuals with NCDs, namely obesity, CVDs and diabetes. ${ }^{42-59}$ While the number of studies regarding culturally adapted IMIs is increasing, it is important to explore these studies' effectiveness and herewith contribute to the development of evidence-based culturally adapted IMIs to promote health behaviours in these underserved populations. Unlike previous reviews, we aim to investigate in a systematic review and meta-analysis whether IMIs can be provided effectively to diverse populations if they are culturally adapted.

\section{Objectives}

The aim of this systematic review and meta-analysis is to investigate the effectiveness of culturally adapted internetbased and mobile-based health promotion interventions. The following questions will be systematically pursued:

1. Are culturally adapted internet-based and mobile-based health promotion interventions effective in the targeted populations?

2. How are culturally adapted IMIs realised and what kind of adaptation features and approaches have been used?

\section{METHODS AND ANALYSIS}

The planned systematic review and meta-analysis has been preregistered at International Prospective Register of Systematic Reviews and follows the Preferred Reporting Items for Systematic Reviews and Meta-Analyses Protocols (PRISMA) 2015 statement. $^{60}$

\section{Eligibility criteria}

Population

The target population should differ in terms of cultural background from the target population that the intervention has been originally developed for.

\section{Interventions}

We will include interventions that were culturally adapted, that is, interventions that were modified in at least one aspect in order to make them more culturally appropriate for the target population. Interventions that have only been translated will not be included. The interventions addressing the following health promotion topics will be included: healthy eating, physical activity, alcohol consumption, sexual health behaviour and smoking cessation. These health promotion areas are endorsed by WHO to improve overall health and fight against the development of NCDs and can be effectively delivered online. ${ }^{17}{ }^{36}$ Health promotion refers to a process which constitutes efforts to enhance overall health and manage its determinants and risk factors. ${ }^{61}$ Furthermore, the intervention has to be delivered predominantly or completely in an internet-based and/or mobile-based setting. By that, we understand the majority $(>50 \%)$ of the content of the intervention has been delivered via the internet and mobile technologies.

\section{Comparators}

Randomised controlled trials (RCTs) comparing a group that receives a culturally adapted IMI with a control group that receives no treatment, treatment as usual, or placebo, as well as with a waitlist or active control group will be included.

\section{Outcomes}

Health behaviour-specific outcomes of interest are:

- For healthy eating interventions: body mass index.

- For physical activity/exercise interventions: time spent exercising (assessed via self-report or mobile/wearable technological devices, eg, pedometers).

- For sexual health behaviour: change in condom use. 
- For smoking cessation: level of smoking.

- For alcohol consumption: level of alcohol consumption.

Cross health behaviour outcomes of interest are:

- Health-related quality of life.

- Self-efficacy.

Outcomes may be reported subjectively (eg, selfreported in questionnaires) or objectively (eg, pedometers, HbA1c (hemoglobin Alc) levels). If more than one measure for an outcome is reported, we will prioritise objective measures over subjective measures.

\section{Study design}

RCTs will be included in order to answer the first research question.

\section{Information sources and search strategy}

Systematic searches were conducted in the following electronic databases to identify eligible studies for the review: Cochrane Central Register of Controlled Trials (CENTRAL), EbscoHost/MEDLINE, Ovid/Embase and EbscoHost/PsychINFO. The search strategy included a combination of keywords and database-specific terms, including the medical subject headings terms that cover culturally adapted IMIs in the field of health promotion interventions (see online supplemental file 1). The search terms also cover the topic of a parallel conducted a systematic review on culturally adapted IMIs for mental health conditions. ${ }^{62}$ We did not add any date limitation to the search and the preliminary search has been conducted on the 26 August 2019. The search will be updated in the process.

\section{Study records}

The results of the search will be uploaded to Covidence, ${ }^{63}$ reference management software. After removal of duplicates, titles and abstracts of the articles will be screened by two independent reviewers (SB and KS) to identify studies with regard to the inclusion criteria. Identified articles will then be reviewed in full text by the two reviewers (SB and KS) according to the inclusion criteria. Any disagreement between the two reviewers regarding eligibility will be resolved by discussion with a third reviewer (HB). Reasons for exclusion will be recorded.

The study selection process is illustrated in a PRISMA flow diagram (see figure 1). ${ }^{64}$

\section{Data items}

Data will be extracted in duplicate by two independent reviewers (SB and KS) in Covidence software. ${ }^{63}$ Extracted data will include publication details, information about the study participants (demographics and cultural background, baseline characteristics), study design, study setting, the country where the intervention was conducted, characteristics of the original and the adapted interventions, and health behaviour-specific and generic outcome measures, the form of cultural adaptation of the intervention, content of the intervention and utilisation of theoretical or evidence-based components of the interventions.

\section{Quality assessment and risk of bias}

The risk of bias will be assessed by two independent reviewers using Cochrane Collaboration's Risk of Bias tool V 2.0 through in following domains: bias arising from the randomisation process, bias from deviations from the intended interventions, bias from missing outcome data, bias due to measurement of the outcome, bias from the selection of the reported results. ${ }^{65}$

\section{Data synthesis}

Meta-analysis

Data will be meta-analytically pooled in case of at least three included studies reporting data on the same outcome. Else findings of included studies will be described descriptively. In order to quantify the heterogeneity, $\mathrm{I}^{2}$ test will be performed for the subgroups, which will be formed based on the intervention outcome of each domain. If heterogeneity is significant, the meta-analysis will be performed using random-effect models. ${ }^{66}$ Review Manager V.5 will be used to conduct the analysis. ${ }^{67}$ In the case of multiple outcome measures reported for one primary outcome, then we will use the objective measure over subjective measures. Postintervention scores of intervention and control groups will be used to calculate the between-group effect sizes of each study. For each outcome domain, the meta-analytical effect will be calculated with standardised mean differences and 95\% CIs for continuous outcomes, whereas for dichotomous outcomes, ORs will be used. The effect sizes will be descriptively categorised according to Cohen's rule of thumb with $\mathrm{d}=0.20$ considered a small effect, $d=0.50$ a medium effect and $d=0.80$ a large effect. ${ }^{68}$ Publication bias will be assessed using funnel plots.

\section{Heterogeneity}

With an assumption of the random-effects model, heterogeneity will be measured with $\mathrm{I}^{2}$ statistics. In order to interpret the results of this, guidelines from the Cochrane Handbook will be used. According to the guidelines, $0 \%-40 \%$ refers to no heterogeneity, $30 \%-60 \%$ moderate, $50 \%-90 \%$ substantial and $75 \%-100 \%$ considerable heterogeneity ${ }^{69}$ In the presence of substantial heterogeneity, we will conduct subgroup analyses to explore possible sources of heterogeneity. If we have sufficient data, subgroup analyses will be conducted across studies based on: population (healthy vs diagnosed), duration of the intervention, technology (internet-based vs app/ mobile-based) and presence of guidance. Sensitivity analyses will be conducted to explore the impact of the risk of bias on study findings. Studies rated as high risk of bias will be excluded and the meta-analysis will be conducted again.

\section{Qualitative synthesis}

A narrative synthesis of all included studies will be conducted, and the relevant characteristics of the studies defined in the Data Items section will be qualitatively 


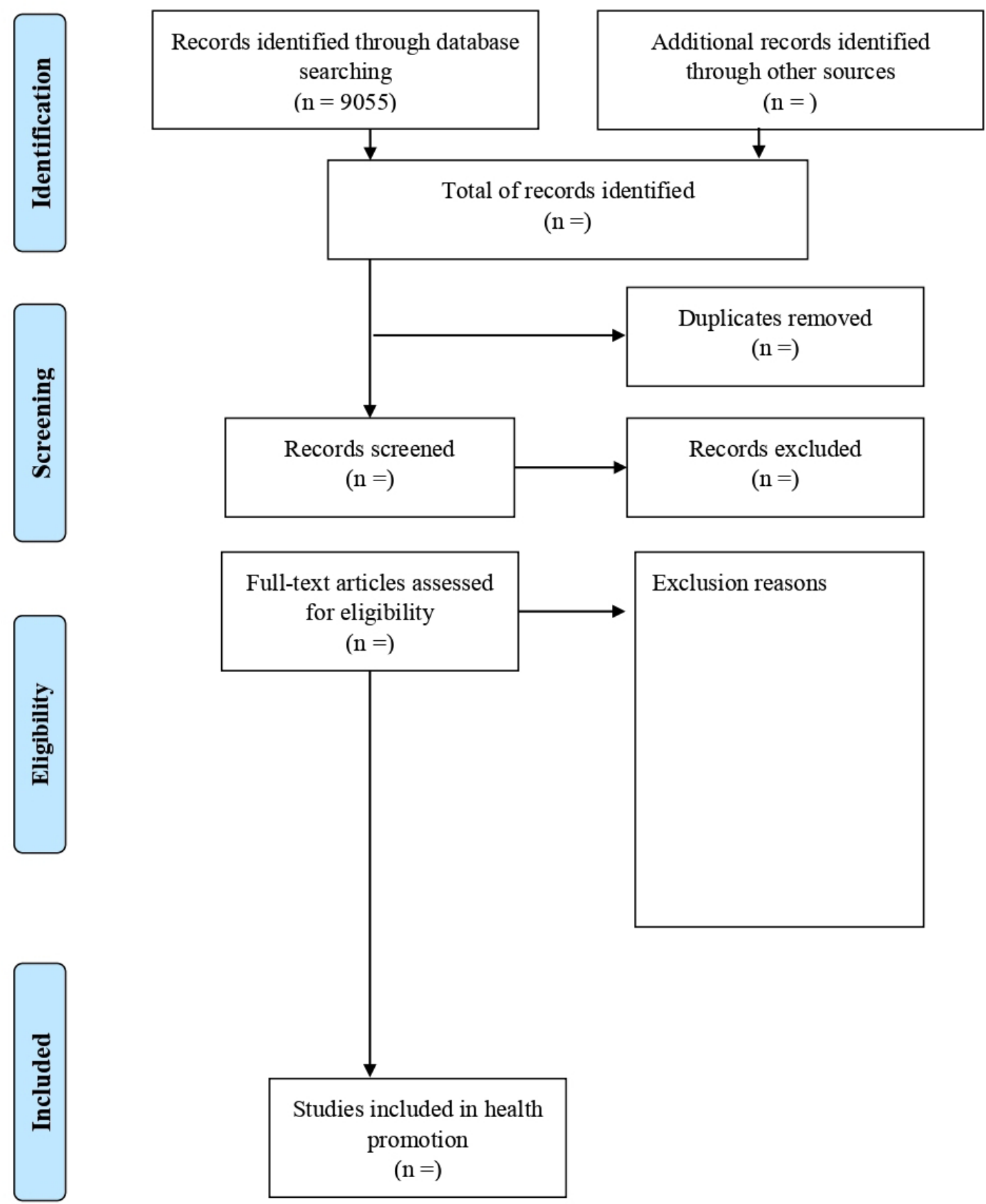

Figure 1 PRISMA flow diagram. Mohar D et al. ${ }^{64}$

described. This synthesis will address the cultural adaptation process, theoretical background and adapted components of the intervention. Moreover, findings of the studies, namely acceptance and adherence rates, will be outlined. A table summarising the objectives, methods, and outcomes of each included study will be provided.

\section{Patient and public involvement}

No patient involved.

\section{ETHICS AND DISSEMINATION}

Since the collected data in this review are based on secondary analyses of published data, no ethical approval is needed. After completing the review and meta-analysis, the results will be published in a peer-reviewed international journal. Moreover, the results will be presented in conferences and workshops. Updates regarding the current status of this review will be available in PROSPERO.

\section{DISCUSSION}

The present planned review is the first to explore the effectiveness of culturally adapted IMIs concerning health promotion interventions and will address a significant lack of research concerning the cultural adaptation of 
IMIs. It is expected to inform future research by providing a summary of the literature in this area and describing the features of existing culturally adapted IMIs. Moreover, this review might communicate practices whether culturally adapting IMIs is a necessary effort to make and may guide future investment. Nonetheless, due to the expected heterogeneity of effects across studies, the findings from this review should be treated with caution.

\section{Twitter Lasse Sander@BosseSander}

Contributors SB, KS, HB and LS conceived the study design. SB, KS and LS developed the search strategy. SB wrote the draft of the manuscript. All authors read, provided feedback and approved the final version. The guarantor of the review is $S B$.

Funding This research has been funded by the SB's Ph.D. scholarship, which is granted by the Ministry of Education in Turkey.

Disclaimer The funder had no involvement in this study and manuscript.

Competing interests HB received consultancy fees, reimbursement of congress attendance and travel costs as well as payments for lectures from Psychotherapy and Psychiatry Associations as well as Psychotherapy Training Institutes in the context of E-Mental-Health topics. He has been the beneficiary of study support (third-party funding) from several public funding organisations. LS reported receiving personal fees from Psychotherapy Training Institutes and clinics in the context of e-mental-health and supervision outside the submitted work.

Patient consent for publication Not required.

Provenance and peer review Not commissioned; externally peer reviewed.

Supplemental material This content has been supplied by the author(s). It has not been vetted by BMJ Publishing Group Limited (BMJ) and may not have been peer-reviewed. Any opinions or recommendations discussed are solely those of the author(s) and are not endorsed by BMJ. BMJ disclaims all liability and responsibility arising from any reliance placed on the content. Where the content includes any translated material, BMJ does not warrant the accuracy and reliability of the translations (including but not limited to local regulations, clinical guidelines, terminology, drug names and drug dosages), and is not responsible for any error and/or omissions arising from translation and adaptation or otherwise.

Open access This is an open access article distributed in accordance with the Creative Commons Attribution Non Commercial (CC BY-NC 4.0) license, which permits others to distribute, remix, adapt, build upon this work non-commercially, and license their derivative works on different terms, provided the original work is properly cited, appropriate credit is given, any changes made indicated, and the use is non-commercial. See: http://creativecommons.org/licenses/by-nc/4.0/.

\section{ORCID iD}

Sumeyye Balci http://orcid.org/0000-0001-8219-6163

\section{REFERENCES}

1 WHO. THE GLOBAL HEALTH OBSERVATORY: Total NCD Mortality, 2016. Available: https://www.who.int/data/gho/data/themes/topics/ indicator-groups/indicator-group-details/GHO/total-ncd-mortality [Accessed 29.01.2020].

2 Di Cesare M, Khang Y-H, Asaria P, et al. Inequalities in noncommunicable diseases and effective responses. The Lancet 2013;381:585-97.

3 Yun K, Hebrank K, Graber LK, et al. High prevalence of chronic noncommunicable conditions among adult refugees: implications for practice and policy. J Community Health 2012;37:1110-8.

4 Montesi L, Caletti MT, Marchesini G. Diabetes in migrants and ethnic minorities in a changing world. World J Diabetes 2016;7:34.

5 Modesti PA, Reboldi G, Cappuccio FP, et al. Panethnic differences in blood pressure in Europe: a systematic review and meta-analysis. PLoS One 2016;11:e0147601.

6 Rambihar VS, Rambihar SP, Rambihar VS. Race, ethnicity, and heart disease: a challenge for cardiology for the 21st century. Am Heart J 2010;159:1-14.

7 Di Cesare M, Khang Y-H, Asaria P, et al. Inequalities in noncommunicable diseases and effective responses. Lancet 2013;381:585-97.
8 Alwan A. Global status report on noncommunicable diseases. World Health Organization, 2011. ISBN:.ISBN:978 9241564229.

9 Benziger CP, Roth GA, Moran AE. The global burden of disease study and the preventable burden of ncd. Glob Heart 2016;11:393-7.

10 Castelli F, Muiesan ML, Migration EHI. Communicable and noncommunicable diseases: are we witnessing a paradigm shift? Springer International Publishing AG, 2018: 17-26.

11 da Silva LS, Cotta RMM, Rosa CdeOB. [Health promotion and primary prevention strategies to fight chronic disease: a systematic review]. Rev Panam Salud Publica 2013;34:343-50.

12 Horton R. Non-Communicable diseases: 2015 to 2025. The Lancet 2013;381:509-10.

13 WHO. Preventing chronic diseases: a vital investment: who global report. World Health Organization, 2005. ISBN: 9241563001. https://apps.who.int/iris/handle/10665/43314

14 United Nations G. Resolution A/RES/65/238: scope, modalities, format and organization of the high-level meeting of the general assembly on the prevention and control of non-communicable diseases, 2011.

15 World Health Assembly, 66. Follow-Up to the political Declaration of the high-level meeting of the general assembly on the prevention and control of non-communicable diseases; 2013. https://apps.who.int/ iris/handle/10665/150161

16 GBD 2017 Risk Factor Collaborators. Global, regional, and national comparative risk assessment of 84 behavioural, environmental and occupational, and metabolic risks or clusters of risks for 195 countries and territories, 1990-2017: a systematic analysis for the global burden of disease study 2017. Lancet 2018;392:1923-94.

17 World Health Organization. Global status report on noncommunicable diseases, 2014. ISBN: . ISBN: 978924156485 4. https://www.who. int/nmh/publications/ncd-status-report-2014/en/

18 Pelikan JM, Dietscher C, Schmied H. Health promotion for NCDs in and by hospitals: a health promoting Hospital perspective. In: Global Handbook on noncommunicable diseases and health promotion. New York, NY: Springer, 2013.

19 Leclerc A, Kaminski M, Lang T. [Closing the gap in a generation: the WHO report on social determinants of health]. Rev Epidemiol Sante Publique 2009;57:227-30.

20 Friel S, Marmot MG. Action on the social determinants of health and health inequities goes global. Annu Rev Public Health 2011;32:225-36

21 WHO regional office for Europe. How health systems can address health inequities linked to migration and ethnicity WHO Reg Off Eur; 2010. https://www.euro.who.int/ data/assets/pdf file/0005/127526/ e94497.pdf [Accessed 07.02.2020]. 9789289002042.

22 Barrera M, Castro FG, Strycker LA, et al. Cultural adaptations of behavioral health interventions: a progress report. J Consult Clin Psychol 2013;81:196-205.

23 Davies AA, Blake C, Dhavan P. Social determinants and risk factors for non-communicable diseases (NCDS) in South Asian migrant populations in Europe. Asia Eur J 2011;8:461-73.

24 Bernal G, Jiménez-Chafey MI, Domenech Rodríguez MM. Cultural adaptation of treatments: a resource for considering culture in evidence-based practice. Prof Psychol 2009;40:361-8.

25 Escoffery $\mathrm{C}$, Lebow-Skelley $\mathrm{E}$, Udelson $\mathrm{H}$, et al. A scoping study of frameworks for adapting public health evidence-based interventions. Transl Behav Med 2019;9:1-10.

26 Bernal G, Sáez-Santiago E. Culturally centered psychosocial interventions. J Community Psychol 2006;34:121-32.

27 Resnicow K, Soler R, Braithwaite RL, et al. Cultural sensitivity in substance use prevention. J Community Psychol 2000;28:271-90.

28 Davidson EM, Liu JJ, Bhopal R, et al. Behavior change interventions to improve the health of racial and ethnic minority populations: a tool kit of adaptation approaches. Milbank Q 2013;91:811-51.

29 Bartholomew LK, Parcel GS, Kok G. Intervention mapping: a process for developing theory- and evidence-based health education programs. Health Educ Behav 1998;25:545-63.

30 Leong FTL, Lee SH. A cultural accommodation model for crosscultural psychotherapy: illustrated with the case of Asian Americans. special issue: culture, race, and ethnicity in psychotherapy. Psychotherapy Theory Research \& Practice 2006;43:410-23.

31 Vandelanotte C, Müller AM, Short CE, et al. Past, present, and future of eHealth and mHealth research to improve physical activity and dietary behaviors. J Nutr Educ Behav 2016;48:219-28.

32 Lustria MLA, Noar SM, Cortese J, et al. A meta-analysis of WebDelivered tailored health behavior change interventions. $J$ Health Commun 2013;18:1039-69.

33 Bennett GG, Steinberg DM, Stoute C, et al. Electronic health (eHealth) interventions for weight management among racial/ethnic minority adults: a systematic review. Obes Rev 2014;15 Suppl 4:146-58. 
34 Webb TL, Joseph J, Yardley L, et al. Using the Internet to promote health behavior change: a systematic review and meta-analysis of the impact of theoretical basis, use of behavior change techniques, and mode of delivery on efficacy. J Med Internet Res 2010;12:e4-18.

35 Bendig E, Bauereiß N, Ebert DD, et al. Internet- based interventions in chronic somatic disease. Dtsch Arztebl Int 2018;115:659-65.

36 Kohl LFM, Crutzen R, de Vries NK. Online prevention aimed at lifestyle behaviors: a systematic review of reviews. J Med Internet Res 2013;15:e146-13.

37 Ryan K, Dockray S, Linehan C. A systematic review of tailored eHealth interventions for weight loss. Digit Health 2019;5

38 Noar SM, Black HG, Pierce LB. Efficacy of computer technologybased HIV prevention interventions: a meta-analysis. AIDS 2009;23:107-15.

39 Hamilton SJ, Mills B, Birch EM, et al. Smartphones in the secondary prevention of cardiovascular disease: a systematic review. BMC Cardiovasc Disord 2018;18:25.

40 Do HP, Tran BX, Le Pham Q, et al. Which eHealth interventions are most effective for smoking cessation? A systematic review. Patient Prefer Adherence 2018;12:2065-84.

41 Müller AM, Alley S, Schoeppe S, et al. The effectiveness of e-\& $\mathrm{mHealth}$ interventions to promote physical activity and healthy diets in developing countries: A systematic review. Int J Behav Nutr Phys Act 2016;13:109.

42 Saleh S, Farah A, Dimassi H, et al. Using mobile health to enhance outcomes of noncommunicable diseases care in rural settings and refugee camps: randomized controlled trial. JMIR Mhealth Uhealth 2018;6:e137.

43 Ni Mhurchu C, Whittaker R, McRobbie H, et al. Feasibility, acceptability and potential effectiveness of a mobile health (mHealth) weight management programme for new Zealand adults. BMC Obes 2014;1:1-10.

44 Zhou W, Chen M, Yuan J, et al. Welltang - A smart phone-based diabetes management application - Improves blood glucose control in Chinese people with diabetes. Diabetes Res Clin Pract 2016;116:105-10.

45 Ramirez M, Wu S. Phone messaging to prompt physical activity and social support among low-income Latino patients with type 2 diabetes: a randomized pilot study. JMIR Diabetes 2017;2:e8.

46 Mimiaga MJ, Thomas B, Biello K, et al. A pilot randomized controlled trial of an integrated In-person and mobile phone delivered counseling and text messaging intervention to reduce HIV transmission risk among male sex workers in Chennai, India. AIDS Behav 2017;21:3172-81.

47 Ybarra ML, Bull SS, Prescott TL, et al. Acceptability and feasibility of CyberSenga: an Internet-based HIV-prevention program for adolescents in Mbarara, Uganda. AIDS Care 2014;26:441-7.

48 Sznitman S, Vanable PA, Carey MP, et al. Using culturally sensitive media messages to reduce HIV-associated sexual behavior in highrisk African American adolescents: results from a randomized trial. $J$ Adolesc Health 2011;49:244-51.

49 Billings DW, Leaf SL, Spencer J, et al. A randomized trial to evaluate the efficacy of a web-based HIV behavioral intervention for high-risk African American women. AIDS Behav 2015;19:1263-74.

50 Albright CL, Steffen AD, Wilkens LR, et al. Effectiveness of a 12-month randomized clinical trial to increase physical activity in multiethnic postpartum women: results from Hawaii's Nā Mikimiki project. Prev Med 2014;69:214-23.
51 Huang S-J, Hung W-C, Shyu M-L, et al. Web-Based intervention to promote physical activity in Taiwanese children. J Pediatr Nurs 2019;45:e35-43

52 Migneault JP, Dedier JJ, Wright JA, et al. A culturally adapted telecommunication system to improve physical activity, diet quality, and medication adherence among hypertensive African-Americans: a randomized controlled trial. Ann Behav Med 2012;43:62-73.

53 Lin M, Mahmooth Z, Dedhia N, et al. Tailored, interactive text messages for enhancing weight loss among African American adults: the TRIMM randomized controlled trial. Am J Med 2015;128:896-904.

54 Brewer LC, Hayes SN, Jenkins SM, et al. Improving cardiovascular health among African-Americans through mobile health: the faith! APP pilot study. J Gen Intern Med 2019;34:1376-8.

55 Staffileno BA, Tangney CC, Fogg L. Favorable outcomes using an eHealth approach to promote physical activity and nutrition among young African American women. J Cardiovasc Nurs 2018;33:62-71.

56 Bender MS, Cooper BA, Park LG, et al. A feasible and efficacious Mobile-Phone based lifestyle intervention for Filipino Americans with type 2 diabetes: randomized controlled trial. JMIR Diabetes 2017;2:e30.

57 Lee HY, Koopmeiners JS, McHugh J, et al. mHealth pilot study: text messaging intervention to promote HPV vaccination. Am J Health Behav 2016;40:67-76.

58 Chandler J, Sox L, Kellam K, et al. Impact of a culturally tailored mHealth medication regimen self-management program upon blood pressure among hypertensive Hispanic adults. Int J Environ Res Public Health 2019;16:1226.

59 Liao Y, Wu Q, Kelly BC, et al. Effectiveness of a text-messagingbased smoking cessation intervention ("Happy Quit") for smoking cessation in China: A randomized controlled trial. PLoS Med 2018;15:e1002713.

60 Shamseer L, Moher D, Clarke M, et al. Preferred reporting items for systematic review and meta-analysis protocols (PRISMA-P) 2015: elaboration and explanation. BMJ 2015;349:g7647:1-25.

61 World Health Organization. Ottawa charter for health promotion first International Conference on health promotion. Health Promotion International 1986;1:405.

62 Spanhel K, Balci S, Baumeister H, et al. Cultural adaptation of Internet- and mobile-based interventions for mental disorders: a systematic review protocol. Syst Rev 2020;9:207.

63 Babineau J. Product review: Covidence (systematic review software). J Can Health Libr Assoc 2014;35:68.

64 Moher D, Liberati A, Tetzlaff J, et al. Preferred reporting items for systematic reviews and meta-analyses: the PRISMA statement. PLoS Med 2009;6:e1000097.

65 Sterne JAC, Savović J, Page MJ, et al. Rob 2: a revised tool for assessing risk of bias in randomised trials. BMJ 2019;366:14898.

66 Higgins JPT, Thompson SG. Quantifying heterogeneity in a metaanalysis. Stat Med 2002;21:1539-58.

67 The Nordic Cochrane centreReview Manager (RevMan) [Computer program] Version 5.3. Copenhagen: The Nordic Cochrane Centre, The Cochrane Collaboration. 2014.

68 Cohen J. Statistical power analysis for the behavioural science. 2nd ed, 1988. ISBN: 978-0805802832.

69 Higgins J, Green S. Cochrane Handbook for systematic reviews of interventions. Version 5.1.0. The Cochrane Collaboration, 2011. www.handbook.cochrane.org 